\title{
Analytic approximation of Volterra's population model
}

\author{
J. BIAZAR AND K. HOSSEINI
}

\begin{abstract}
In this paper, the Volterra's population model is studied for population growth of a species within a closed system. Modified Adomian decomposition method (MADM) in conjunction with Pade technique is formally proposed to obtain an analytic approximation for the solution of the model, which is a nonlinear intgro-differential equation. The results of the method are compared with the existing exact results, confirming the accuracy and the efficiency of the proposed approach.
\end{abstract}

Keywords: Volterra's population model; Nonlinear intgro-differential equation; Modified Adomian decomposition method; Pade technique; Analytic approximation.

Mathematics Subject Classification 2010: 45J05; 34K28

\section{INTRODUCTION}

The Volterra model for population growth of a species within a closed system is known as follows [1,2]

$$
\frac{d p}{d \hat{t}}=a p-b p^{2}-c p \int_{0}^{\hat{t}} p(x) d x, \quad p(0)=p_{0},
$$

where $a>0$ is the birth rate coefficient, $b>0$ is the crowding coefficient, $c>0$ is the toxicity coefficient, $p_{0}$ is the initial population, and $p=p(\hat{t})$ indicates the population at time $\hat{t}$. By introducing the non-dimensional variables

$$
t=\frac{\hat{t}}{b / c}, \quad u=\frac{p}{a / b},
$$

Eq. (1) can be displayed in the following form

$$
\kappa \frac{d u}{d t}=u-u^{2}-u \int_{0}^{t} u(x) d x, \quad u(0)=u_{0},
$$


where $\kappa=c / a b$ is a non-dimensional parameter. It can be shown that the only equilibrium solution to Eq. (2) is the trivial solution $u(t)=0$. Moreover, the following analytical solution [2]

$$
u(t)=u_{0} \exp \left(\frac{1}{\kappa} \int_{0}^{t}\left(1-u(\tau)-\int_{0}^{\tau} u(x) d x\right) d \tau\right)
$$

demonstrates that $u(t)>0$ for all $t$ if $u_{0}>0$.

Although a closed form solution has been reported in [1,3], it was illustrated that the closed form solution cannot yield any insight into the behavior of the population evolution [1]. In recent years, a number of methods have been used for the solution of the Volterra's population model (2). Some of them are listed, here. Dehghan and Shahini used a pseudospectral approach based on the rational Legendre and rational Chebyshev functions [4]. Mohyud-Din et al. adopted a combination of homotopy perturbation method and Pade technique [5]. Wazwaz employed Adomian decomposition method in conjunction with Pade technique [6]. Al-Khaled applied Sinc-Galerkin method [7]. Ramezani et al. utilized composite spectral functions [8]. Parand et al. implemented two collocation approaches based on the radial basis functions [9]. Parand et al. also employed the collocation method with sinc and rational Legendre functions [10]. El-Nahhas used homotopy analysis method [11]. Tabatabaei and Gunerhan utilized differential transform method [12]. The reader is also referred to [13-17]. In this paper, a new and effective modification of ADM in conjunction with Pade technique is formally proposed to construct an approximate solution of the Volterra's population model.

This article is organized as follows: In Section 2, the basic ideas of the method are presented, and then, some Theorems are given. In Section 3, the method is utilized for the solution of the Volterra's population model. In Section 4, the results of the method are compared with the existing exact results. Conclusions are provided in the last section. 


\section{MODIFIED ADOMIAN DECOMPOSITION METHOD}

Consider a nonlinear equation as follows

$$
\frac{d u}{d t}+R(u)+N(u)=f(t)
$$

subject to the following initial condition

$$
u(0)=\xi
$$

where $R$ is a linear operator, $N$ is a nonlinear operator, and $f(t)$ is a known function. It is assumed that the solution of Eq. (3) can be expressed by a series, say

$$
u(t)=\sum_{n=0}^{+\infty} u_{n}(t)
$$

and the nonlinear term $N(u)$ can be presented as the following series

$$
N(u)=\sum_{n=0}^{+\infty} A_{n}\left(u_{0}, u_{1}, \ldots, u_{n}\right),
$$

where $A_{n}, n=0,1, \ldots$ are called the Adomian polynomials and are defined by [18]

$$
A_{n}= \begin{cases}N\left(u_{0}\right) & n=0, \\ \frac{1}{n} \sum_{i=0}^{n-1}(i+1) u_{i+1} \frac{d A_{n-1-i}}{d u_{0}}, & n=1,2, \ldots\end{cases}
$$

Applying $L^{-1}=\int_{0}^{t}() d$.$t to both sides of Eq. (3) and considering (4) and (5), leads to$

$$
\sum_{n=0}^{+\infty} u_{n}=u(0)+L^{-1}(f(t))-L^{-1}\left(R\left(\sum_{n=0}^{+\infty} u_{n}\right)+\sum_{n=0}^{+\infty} A_{n}\left(u_{0}, u_{1}, \ldots, u_{n}\right)\right) .
$$

We rewrite (6) as follows [19-21]

$$
\begin{aligned}
\sum_{n=0}^{+\infty} u_{n}= & u(0)+L^{-1}\left(\sum_{n=0}^{+\infty} a_{n} t^{n}\right)-p L^{-1}\left(\sum_{n=0}^{+\infty} a_{n} t^{n}\right) \\
& +L^{-1}(f(t))-L^{-1}\left(R\left(\sum_{n=0}^{+\infty} u_{n}\right)+\sum_{n=0}^{+\infty} A_{n}\left(u_{0}, u_{1}, \ldots, u_{n}\right)\right),
\end{aligned}
$$


where $p$ is an artificial parameter and $a_{i}, i=0,1, \ldots$ are unknown coefficients. Now, we define

$$
\begin{aligned}
& u_{0}=\xi+L^{-1}\left(\sum_{n=0}^{+\infty} a_{n} t^{n}\right), \\
& u_{1}=L^{-1}(f(t))-p L^{-1}\left(\sum_{n=0}^{+\infty} a_{n} t^{n}\right)-L^{-1}\left(R\left(u_{0}\right)+A_{0}\left(u_{0}\right)\right), \\
& u_{n+1}=-L^{-1}\left(R\left(u_{n}\right)+A_{n}\left(u_{0}, u_{1}, \ldots, u_{n}\right)\right), \quad n=1,2, \ldots
\end{aligned}
$$

To avoid evaluation of $A_{n}, n=1,2, \ldots$, let compute $a_{i}, i=0,1, \ldots$ such that $u_{1}=0$. This results in

$$
u_{2}=u_{3}=\cdots=0 \text {. }
$$

Setting $p=1$, provides the solution of Eq. (3) with initial condition as

$$
u(t)=\xi+L^{-1}\left(\sum_{n=0}^{+\infty} a_{n} t^{n}\right)
$$

NOTE 1. It should be stated that the superiority of the method over traditional $\mathrm{ADM}$ is that in some problems, especially singular ones, the method provides a solution in the form of a convergent series, whereas the traditional ADM fails [21].

THEOREM 1. If $u_{1}=0$, then

$$
u_{n+1}=-L^{-1}\left(R\left(u_{n}\right)+A_{n}\left(u_{0}, u_{1}, \ldots, u_{n}\right)\right)=0, \quad n=1,2, \ldots .
$$

PROOF. From the following definition of Adomian polynomials

$$
A_{n}=\frac{1}{n} \sum_{i=0}^{n-1}(i+1) u_{i+1} \frac{d A_{n-1-i}}{d u_{0}}, \quad n=1,2, \ldots
$$

and knowing that $R$ is a linear operator, the proof is clear.

THEOREM 2. The equation $u_{1}=0$ is equivalent to Eq. (3), when $p=1$. 
Proof. Applying the operator $L$ to both sides of $u_{1}=0$, yields

$$
f(t)-L\left(p L^{-1}\left(\sum_{n=0}^{+\infty} a_{n} t^{n}\right)\right)-R\left(u_{0}\right)-N\left(u_{0}\right)=0 .
$$

But

$$
L=\frac{d}{d t}, \quad u_{0}=\xi+L^{-1}\left(\sum_{n=0}^{+\infty} a_{n} t^{n}\right)
$$

Therefore

$$
\frac{d}{d t}\left(p\left(u_{0}-\xi\right)\right)+R\left(u_{0}\right)+N\left(u_{0}\right)=f(t), \quad \xi=\text { constant }
$$

Now, if we set $p=1$, then

$$
\frac{d}{d t}\left(u_{0}\right)+R\left(u_{0}\right)+N\left(u_{0}\right)=f(t)
$$

which is the original Eq. (3).

THEOREM 3. There exists a number $\rho \in[0,+\infty]$, defined by

$$
\rho=\frac{1}{\lim _{k \rightarrow+\infty} \sup \left|a_{k}\right|^{1 / k}}
$$

such that the series solution (7), converges absolutely, if $|t|<\rho$, and diverges, if $|t|>\rho$

PROOF. See [22]. 


\section{SOLUTION OF VOLTERRA'S POPULATION MODEL BY MODIFIED ADM}

By applying $L^{-1}=\int_{0}^{t}() d$.$t to both sides of Eq. (2) and considering$

$$
u(t)=\sum_{n=0}^{+\infty} u_{n}(t), \quad u^{2}(t)=\sum_{n=0}^{+\infty} A_{n}(t), \quad u(x) \mu(t)=\sum_{n=0}^{+\infty} B_{n}(x, t),
$$

one obtains

$$
\sum_{n=0}^{+\infty} u_{n}(t)=u(0)+\frac{1}{\kappa} L^{-1}\left(\sum_{n=0}^{+\infty} u_{n}(t)-\sum_{n=0}^{+\infty} A_{n}(t)-\int_{0}^{t} \sum_{n=0}^{+\infty} B_{n}(x, t) d x\right) .
$$

The first few Adomian polynomials are as follows

$$
\begin{aligned}
& A_{0}(t)=u_{0}^{2}(t), \quad B_{0}(x, t)=u_{0}(x) u_{0}(t) \\
& A_{1}(t)=2 u_{0}(t) u_{1}(t), \quad B_{1}(x, t)=u_{0}(x) u_{1}(t)+u_{1}(x) u_{0}(t),
\end{aligned}
$$

We rewrite (8) as follows

$$
\begin{aligned}
\sum_{n=0}^{+\infty} u_{n}(t)= & u(0)+L^{-1}\left(\sum_{n=0}^{+\infty} a_{n} t^{n}\right)-p L^{-1}\left(\sum_{n=0}^{+\infty} a_{n} t^{n}\right) \\
& +\frac{1}{\kappa} L^{-1}\left(\sum_{n=0}^{+\infty} u_{n}(t)-\sum_{n=0}^{+\infty} A_{n}(t)-\int_{0}^{t} \sum_{n=0}^{+\infty} B_{n}(x, t) d x\right),
\end{aligned}
$$

where $p$ is an artificial parameter and $a_{i}, i=0,1, \ldots$ are unknown coefficients. We now define

$$
\begin{aligned}
& u_{0}(t)=u(0)+L^{-1}\left(\sum_{n=0}^{+\infty} a_{n} t^{n}\right)=u_{0}+a_{0} t+\frac{1}{2} a_{1} t^{2}+\frac{1}{3} a_{2} t^{3}+\cdots, \\
& u_{1}(t)=-p L^{-1}\left(\sum_{n=0}^{+\infty} a_{n} t^{n}\right)+\frac{1}{\kappa} L^{-1}\left(u_{0}(t)-A_{0}(t)-\int_{0}^{t} B_{0}(x, t) d x\right), \\
& u_{n+1}(t)=\frac{1}{\kappa} L^{-1}\left(u_{n}(t)-A_{n}(t)-\int_{0}^{t} B_{n}(x, t) d x\right), \quad n=1,2, \ldots
\end{aligned}
$$


To avoid calculation of $A_{n}(t)$ and $B_{n}(x, t), n=1,2, \ldots$, let us determine $a_{i}, i=0,1, \ldots$ such that $u_{1}(t)=0$. Therefore

$$
u_{1}(t)=\left(\frac{u_{0}}{\kappa}-\frac{u_{0}^{2}}{\kappa}-p a_{0}\right) t+\left(\frac{1}{2 \kappa} a_{0}-\frac{u_{0}}{\kappa} a_{0}-\frac{u_{0}^{2}}{2 \kappa}-\frac{1}{2} p a_{1}\right) t^{2}+\cdots=0
$$

It can be shown that

$$
a_{0}=-\frac{u_{0}\left(u_{0}-1\right)}{\kappa p}, a_{1}=-\frac{u_{0}\left(p \kappa u_{0}-2 u_{0}^{2}+3 u_{0}-1\right)}{\kappa^{2} p^{2}}, \cdots .
$$

Setting $p=1$, yields the solution of the Volterra population model as follows

$$
u(t)=u_{0}-\frac{u_{0}\left(u_{0}-1\right)}{\kappa} t-\frac{u_{0}\left(\kappa u_{0}-2 u_{0}^{2}+3 u_{0}-1\right)}{2 \kappa^{2}} t^{2}+\cdots
$$

It should be noted that for $u_{0}=0.1$ and $\kappa=0.1$, the solution $u(t)$ is reduced to

$$
\begin{aligned}
u(t)= & 0.1+0.9 t+3.55 t^{2}+6.316666668 t^{3}-5.537499993 t^{4}-63.70916666 t^{5} \\
& -156.0804167 t^{6}-18.47323414 t^{7}+1056.288569 t^{8}+\cdots
\end{aligned}
$$

that is the same as those reported in $[5,6]$.

THEOREM 4. Volterra's population model is equivalent to a nonlinear ordinary differential equation of the form

$$
\kappa y^{\prime \prime}(t)-y^{\prime}(t)+\left(y^{\prime}(t)\right)^{2}+y(t) y^{\prime}(t)=0, \quad y(0)=0, \quad y^{\prime}(0)=u_{0} .
$$

\section{PROOF. Setting}

$$
y(t)=\int_{0}^{t} u(x) d x
$$

yields

$$
y^{\prime}(t)=u(t), \quad y^{\prime \prime}(t)=u^{\prime}(t)
$$


Substituting (11) and (12) into Eq. (2) leads to

$$
\kappa y^{\prime \prime}(t)-y^{\prime}(t)+\left(y^{\prime}(t)\right)^{2}+y(t) y^{\prime}(t)=0, \quad y(0)=0, \quad y^{\prime}(0)=u_{0} .
$$

This completes the proof.

NOTE 2. It is easy to show that applying the method to nonlinear ordinary differential equation (10) results in the previous solution (9). It is shown in Appendix A.

\section{RESULTS AND DISCUSSION}

Let us consider Eq. (2) with initial condition $u(0)=0.1$ and $\kappa=0.1$. It was shown in the previous section, for such a problem using modified Adomian decomposition method the following analytic solution can be obtained

$$
\begin{aligned}
u(t)= & 0.1+0.9 t+3.55 t^{2}+6.316666668 t^{3}-5.537499993 t^{4}-63.70916666 t^{5} \\
& -156.0804166 t^{6}-18.47323414 t^{7}+1056.288569 t^{8}+\cdots
\end{aligned}
$$

As we know, another way of representing the behavior of $u(t)$ is to use Pade approximants. Pade approximants provide results with no greater error bounds than approximation by polynomials [6]. The Pade approximant [4/4] of $u(t)$ is as follows

$[4 / 4]=\frac{0.1+0.4687931825 t+0.9249573976 t^{2}+0.9231294949 t^{3}+0.4004235135 t^{4}}{1-4.312068175 t+12.55818755 t^{2}-13.88063941 t^{3}+10.86830490 t^{4}}$

Using the maximize function of the Maple package, it can be shown that

$$
u_{\max }=0.7651130842 \text {, }
$$

occurs at

$$
t_{\text {critical }}=0.4645100343 \text {. }
$$


Figure 1 indicates the Pade approximant [4/4] of $u(t)$ versus independent variable $t$. As it is observed, the graph demonstrates the rapid rise along the logistic curve followed by the slow exponential decay after reaching the maximum point. Figure 2 presents the Pade approximant [4/4] of $u(t)$ for $u(0)=0.1$ and different values of $\kappa$. From this Figure, it is clear that as $\kappa$ increases, $u(t)$ decreases, whereas the exponential decay increases. Table 1 shows a comparison between the exact values of $u_{\max }$ given by [2]

$$
u_{\max }=1+\kappa \ln \frac{\kappa}{1+\kappa-u_{0}},
$$

and the results of the present method. From Table 1, the accuracy and the efficiency of the proposed method in solving the Volterra's population model is evident. It should be mentioned that the results can be improved by calculating further terms of solution series.

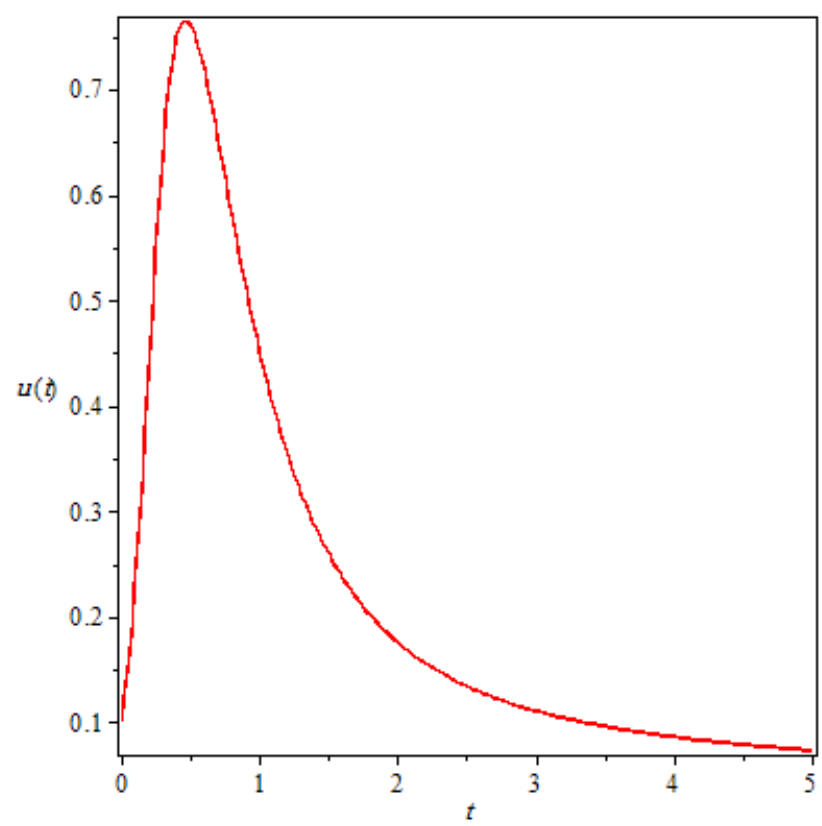

Figure 1: Pade approximant [4/4] of $u(t)$ for $u(0)=0.1$ and $\kappa=0.1$ versus $t$. 


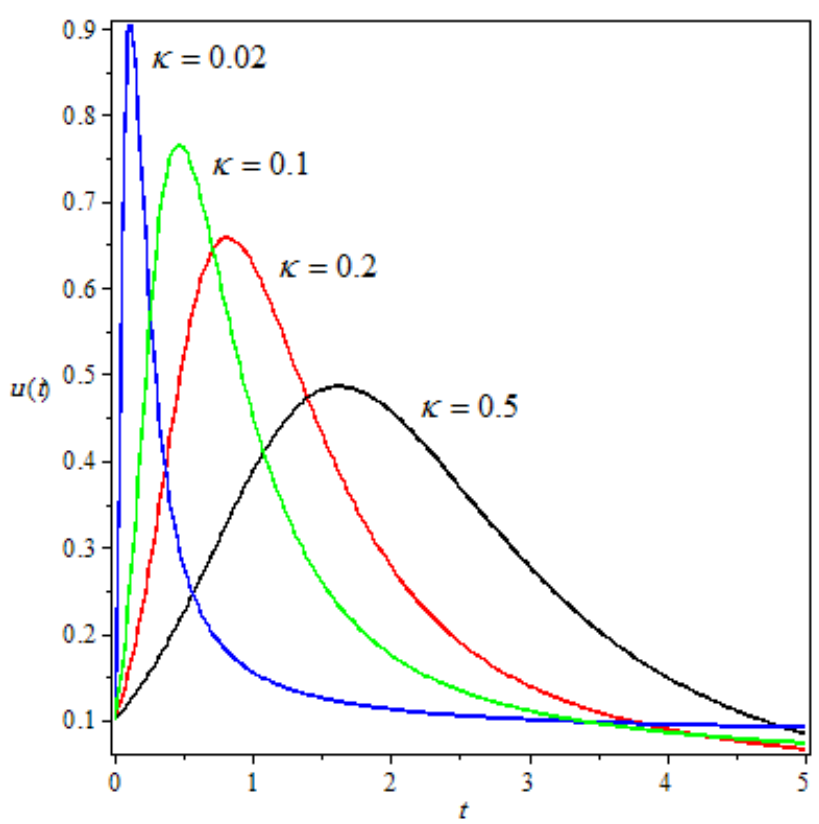

Figure 2: Pade approximant [4/4] of $u(t)$ for $u(0)=0.1$ and $\kappa=0.02,0.1,0.2$, and 0.5 .

Table 1: Results of the present method in comparison to the exact values of $u_{\max }$ $(u(0)=0.1)$.

\begin{tabular}{llll}
\hline$\kappa$ & Critical $t$ & Approximation $u_{\max }$ & Exact $u_{\max }$ \\
0.02 & 0.1118531315 & 0.9038380646 & 0.9234271721 \\
0.1 & 0.4645100343 & 0.7651130842 & 0.7697414907 \\
0.2 & 0.8168581260 & 0.6579123129 & 0.6590503816 \\
0.5 & 1.626662229 & 0.4852823500 & 0.4851902915 \\
\hline
\end{tabular}




\section{CONCLUSION}

A nonlinear integro-differential equation called the Volterra's population model has been studied successfully. A combination of modified Adomian decomposition method introduced by the authors and Pade technique has been used to extract an analytic approximation for the solution of the model. The method has also been examined on an equivalent nonlinear ordinary differential equation, confirming the previous results obtained. It was observed that

1. As $\kappa$ increases, $u(t)$ decreases, whereas the exponential decay increases.

2. The results of the present method are in agreement with the exact values of $u_{\max }$.

It is worth mentioning that the proposed method only requires the calculation of the first Adomian polynomial and suggests a promising technique for solving nonlinear problems.

\section{Appendix A}

In a manner similar to that described in Section 2, we can define

$$
\begin{aligned}
& y_{0}=y(0)+y^{\prime}(0) t+L^{-1}\left(\sum_{n=0}^{+\infty} a_{n} t^{n}\right)=u_{0} t+\frac{1}{2} a_{0} t^{2}+\frac{1}{6} a_{1} t^{3}+\frac{1}{12} a_{2} t^{4}+\cdots, \\
& y_{1}=-p L^{-1}\left(\sum_{n=0}^{+\infty} a_{n} t^{n}\right)+\frac{1}{\kappa} L^{-1}\left(y_{0}^{\prime}-\left(y_{0}^{\prime}\right)^{2}-y_{0} y_{0}^{\prime}\right),
\end{aligned}
$$

where $L^{-1}=\int_{0}^{t} \int_{0}^{t}() d t d$.$t . Now, setting y_{1}=0$, leads to

$$
y_{1}=\left(\frac{u_{0}}{2 \kappa}-\frac{u_{0}^{2}}{2 \kappa}-\frac{1}{2} p a_{0}\right) t^{2}+\left(\frac{1}{6 \kappa} a_{0}-\frac{u_{0}}{3 \kappa} a_{0}-\frac{u_{0}^{2}}{6 \kappa}-\frac{1}{6} p a_{1}\right) t^{3}+\cdots=0 .
$$


It can be shown that

$$
a_{0}=-\frac{u_{0}\left(u_{0}-1\right)}{\kappa p}, \quad a_{1}=-\frac{u_{0}\left(p \kappa u_{0}-2 u_{0}^{2}+3 u_{0}-1\right)}{\kappa^{2} p^{2}}, \cdots .
$$

Setting $p=1$, gives the solution of Eq. (10) as follows

$$
y(t)=u_{0} t-\frac{u_{0}\left(u_{0}-1\right)}{2 \kappa} t^{2}-\frac{u_{0}\left(\kappa u_{0}-2 u_{0}^{2}+3 u_{0}-1\right)}{6 \kappa^{2}} t^{3}+\cdots
$$

Since $u(t)=y^{\prime}(t)$, therefore

$$
u(t)=u_{0}-\frac{u_{0}\left(u_{0}-1\right)}{\kappa} t-\frac{u_{0}\left(\kappa u_{0}-2 u_{0}^{2}+3 u_{0}-1\right)}{2 \kappa^{2}} t^{2}+\cdots,
$$

that is the same as that obtained in the previous section.

\section{REFERENCES}

1. Scudo F (1971) Vito Volterra and theoretical ecology. Theor Popul Biol 2:1-23

2. TeBeest K (1997) Numerical and analytical solutions of Volterra's population model. SIAM Rev 39:484-493

3. Small R (1983) Population growth in a closed system. SIAM Rev 25:93-95

4. Dehghan M, Shahini M (2015) Rational pseudospectral approximation to the solution of a nonlinear integro-differential equation arising in modeling of the population growth. Appl Math Modelling 39:5521-5530

5. Mohyud-Din ST, Yıldırım A, Gulkanat Y (2010) Analytical solution of Volterra's population model. J King Saud Univ 22:247-250

6. Wazwaz AM (1999) Analytical approximations and Pade approximants for Volterra's population model. Appl Math Comput 100:13-25

7. Al-Khaled K (2005) Numerical approximations for population growth models. Appl Math Comput $160: 865-873$

8. Ramezani M, Razzaghi M, Dehghan M (2007) Composite spectral functions for solving Volterra's population model. Chaos Solitons Fract 34:588-593

9. Parand K, Abbasbandy S, Kazem S, Rad JA (2011) A novel application of radial basis functions for solving a model of first-order integro-ordinary differential equation. Commun Nonlinear Sci Numer Simulat 16:4250-4258 
10. Parand K, Delafkar Z, Pakniat N, Pirkhedri A, Kazemnasab Haji M (2011) Collocation method using sinc and rational Legendre functions for solving Volterra's population model. Commun Nonlinear Sci Numer Simulat 16:1811-1819

11. El-Nahhas A (2007) Analytic approximations for Volterra's population equation. Proc Pakistan Acad Sci 44:255-261

12. Tabatabaei K, Gunerhan E (2015) The differential transform method for solving Volterra's population model. AASCIT Commun 2:262-267

13. $\mathrm{Xu} \mathrm{H} \mathrm{(2009)} \mathrm{Analytical} \mathrm{approximations} \mathrm{for} \mathrm{a} \mathrm{population} \mathrm{growth} \mathrm{model} \mathrm{with} \mathrm{fractional} \mathrm{order.}$ Commun Nonlinear Sci Numer Simulat 14:1978-1983

14. Babolian E, Biazar J (2003) Solving concrete examples by Adomian method. Appl Math Comput 135:161-167

15. Parand K, Hossayni SA, Rad JA (2016) An operation matrix method based on Bernstein polynomials for Riccati differential equation and Volterra population model. Appl Math Modelling 40:993-1011

16. Parand K, Razzaghi M (2004) Rational Chebyshev tau method for solving Volterra's population model. Appl Math Comput 149:893-900

17. Yuzbasi S (2013) A numerical approximation for Volterra's population growth model with fractional order. Appl Math Modelling 37:3216-3227

18. Duan S (2011) Convenient analytic recurrence algorithms for the Adomian polynomials. Appl Math Comput 217:6337-6348

19. Aminikhah H, Biazar J (2009) A new HPM for ordinary differential equations. Numer Methods Partial Differ Equ 26:480-489

20. Hosseini K, Biazar J, Ansari R, Gholamin P (2012) A new algorithm for solving differential equations. Math Methods Appl Sci 35:993-999

21. Biazar J, Hosseini K (2016) A modified Adomian decomposition method for singular initial value Emden-Fowler type equations. Int J Appl Math Research 5:69-72

22. Pedersen S (2015) From calculus to analysis. Springer, Switzerland

\author{
J. Biazar \\ Department of Applied Mathematics, \\ Faculty of Mathematical Science, University of Guilan, Rasht, Iran \\ and \\ Department of Mathematics, \\ Rasht Branch, Islamic Azad University, Rasht, Iran \\ K. Hosseini \\ Department of Mathematics, \\ Rasht Branch, Islamic Azad University, Rasht, Iran \\ e-mail address: kamyar_hosseini@yahoo.com
}

